\title{
The Effect of Nonlinear Transport on Beam Halo Formation
}

\author{
Kiran Sonnad and J. R. Cary \\ CIPS and Department of Physics, University of Colorado, Boulder
}

\section{Abstract}

Halo formation is an important issue in intense charged particle beams because the loss of halo particles can lead to radioactivation of the accelerator. In this paper, we investigate the influence of the addition of nonlinear terms to the linear focusing as a possible mechanism to control halo formation. With nonlinear focusing, the core oscillations can no longer be described by an envelope equation. The use of more self consistent models like particle-in-cell codes become necessary. Using a one dimensional particlein cell code, we make comparisons between beams with linear and nonlinear focusing. We also discuss the physical parameters that are set before launching the beam in order to make such comparisons.

\section{INTRODUCTION}

High current accelerators have been proposed for a variety of applications such as high-energy and nuclear physics research, accelerator production of tritium, heavy ion fusion, high-intensity microwave sources etc. This has made necessary the need for the study of halo formation in such beams. A consequence of beam halos is the loss of particles to the system which leads to radioactivation of the machine.

Using an analytic model with a round beam in a uniform linear focusing channel, it was shown by Gluckstern [1] that some particles can acquire high amplitudes of oscillation due to a resonant interaction between the particles and the oscillating beam core. These particles travel into the halo region. The resonant particles had an oscillation frequency which was one half the envelope frequency. It is not only important to better understand beam halo formation but also investigate methods to mitigate them. We have proposed the introduction of nonlinear components to external focusing as a possible method to suppress halo formation. The motivation for this is to investigate the possibility that particles acquiring large amplitudes of oscillation will detune from the resonant interaction as a result of their modified oscillations.

In this paper, we present results obtained from a onedimensional particle-in-cell code. We make a comparison between beams traveling through channels with linear and nonlinear focusing respectively.

\section{PARAMETERS DESCRIBING THE BEAMS}

We assume that the beam is subjected to a continuous focusing force given by

$$
F=-K_{1} x-K_{3} x^{3}
$$

In the linear case, $K_{3}=0$ and we denote $K_{1}$ by $K_{o}$ for this case for easy identification. In addition to this force, the particles will experience a space charge force. We use a round beam where only the breathing mode of the envelope is excited. In the presence liner focusing, the beam envelope can be described by the following equation.

$$
\frac{d^{2} R}{d^{s}}+K_{o} R-\frac{\epsilon^{2}}{R^{3}}-\frac{Q}{R}=0
$$

where the focusing is represented by $K_{o} R, \epsilon=$ $4 \sqrt{\overline{r^{2}} \overline{r^{\prime 2}}-{\overline{r^{\prime} r}}^{2}}$ is the emittance. $\mathrm{Q}$ is the space charge perveance given by $Q=\frac{q I}{2 \pi \epsilon m c^{3} 3 \beta^{3} \gamma^{3}}$. $\mathrm{q}$ is the charge, $\mathrm{m}$ is the mass and $\beta c$ is the axial velocity of the particles respectively. $\gamma$ is the relativistic Lorentz factor. Using this equation, we can obtain an expression for the matched radius by setting $d^{2} R / d s^{2}=0$. The matched beam radius is given by $R_{o}=\left(\epsilon / K_{o}\right)\left[u+\sqrt{1+u^{2}}\right]$, where $u=Q / \epsilon K_{o}$. The initial mismatch $\mathrm{M}$ is then given by $R_{i} / R_{o}$ where $R_{i}$ is the initial radius.

It is difficult to define the parameters that can decide whether two beams are equivalent or not since the linear and nonlinear forces cannot be equated. However it is important that certain conditions are set which prevents one to arbitrarily increase the focusing of one kind before making a comparison. Such results could be misleading. For example, one may obtain better confinement for one case simply because the overall focusing is stronger rather than the different nature of the focusing.

The values of $K_{o}, K_{1}$ and $K_{3}$ are chosen in such a way that both the focusing channels allow the same amount of perveance for a cold, equilibrium beam with the same radius. By balancing the space charge force with the focusing force, we can obtain the resulting charge distributions. In the linear focusing case, this becomes a KV beam with uniform charge distribution within the core and zero charge outside. In the nonlinear focusing case, the equilibrium charge density has an additional quadratic term. This leads to the condition

$$
Q=K_{1} R_{o}^{2}+K_{3} R_{o}^{4}
$$

where Q has the same value for both the $K_{3}=0$ and $K_{3} \neq 0$ cases. We then loosely extend this condition to 
any charge distribution, and replace $R_{o}$ by the initial rms size of the beam.

To estimate equivalent tune depressions, we use the expression for rms tune depression given by Gluckstern et.al [2]. When extended to the additional focusing term, this takes the form,

$$
\eta_{r m s}^{2}=\frac{m \overline{x^{\prime 2}}}{K_{1} \overline{x^{2}}+K_{3} \overline{x^{4}}} .
$$

Once again, we let this parameter remain the same for the $K_{3}=0$ and the $K_{3} \neq 0$ case in order to make a comparison.

\section{PRESENTATION OF RESULTS}

In the nonlinear focusing case, the breathing mode cannot be described by an envelope equation. This calls for the need for a self consistent method of calculation like a particle-in-cell code. We use a one dimensional radial particle-in-cell code for both the focusing channels. Figs.14 show phase space distributions of 50000 particles obtained from this code. The particles are chosen along the $\mathrm{x}-$ axis simply because this has been the convention followed in this research in the past. However, we do a mapping to cylindrical coordinates to exploit the azimuthal symmetry while calculating the fields using Gauss's law at each time step. The focusing forces are chosen so that $K_{1} / K_{o}=0.7$ and $K_{3} \overline{r^{2}} / K_{o}=0.3$ before the launch of the beam. This makes it consistent with the condition mentioned in the previous section. The initial mismatch $\mathrm{M}$ has been set to 0.7 for all the linear focusing cases. The initial particle distribution is Gaussian in velocity and position space and is identical for the linear and the corresponding nonlinear cases. The beams are propagated for 40 undepressed linear oscillations. That is, $\sqrt{K_{o}} S / 2 \pi=40$ where $\mathrm{S}$ is the distance of propagation. Figs. 1 and 2 show the results for linear and nonlinear focusing respectively, both with an rms tune depression of 0.92 , an emittance dominated beam. Figs. 3 and 4 have an rms tune depression of 0.311 which is a space charge dominated beam for linear and nonlinear focusing respectively.

The results of the simulations indicate that the additional nonlinear component in focussing leads to better confinement of the beam. While the reason for this is not clear as yet, there could be various possibilities. We know that the particles in the nonlinear case do not oscillate in phase or with the same velocities even within the core. This causes heating of the beam. As a result, we see that the nonlinearly focussed beams are more spread out in velocity when compared to only the core of the linearly focussed beams. This causes a damping of the core oscillations as shown in figs 5 and 6 , which could be a candidate for suppressing the halo. It is also equally likely that the modified oscillations of the particles itself can cause them to detune from the resonance condition present in the linear focusing case.

There seems to be a general broadening of the beam even before the halo particles are produced. This could be be- cause the initial distribution is not a function of the Hamiltonian, ie, they are not stationary solutions of the Vlasov equation. Such a nonequlibrium distribution leads to an initial remixing process which leads to beam broadening. In order to mask this effect, one needs to start with a distribution that is near a stationary state according to Vlasov's equation [2].

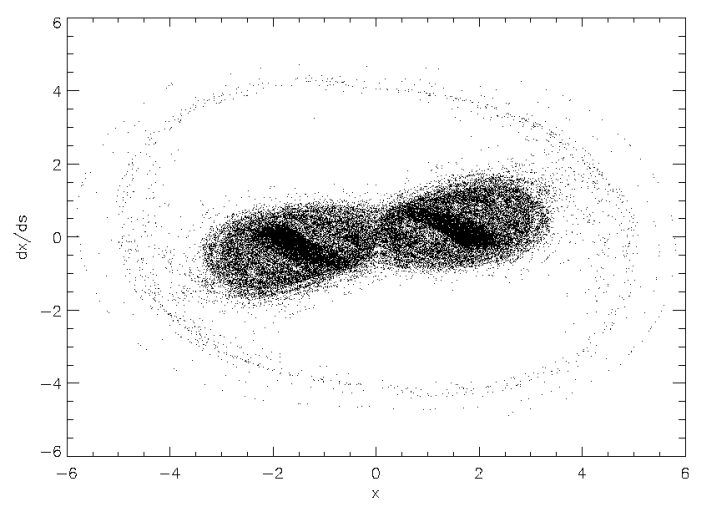

Figure 1: linear force, $\eta_{r m s}=0.92$

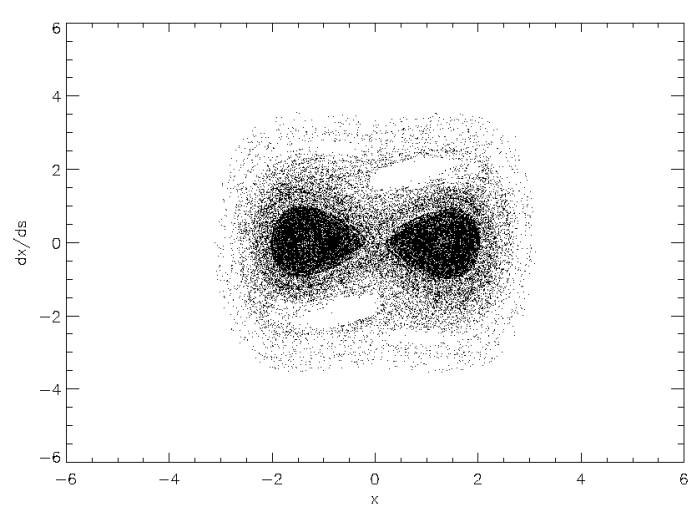

Figure 2: linear force, $\eta_{r m s}=0.92$

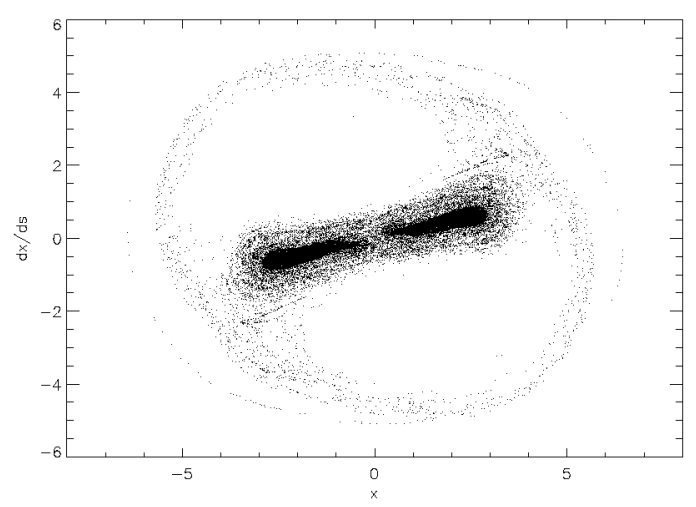

Figure 3: linear force, $\eta_{r m s}=0.31$ 


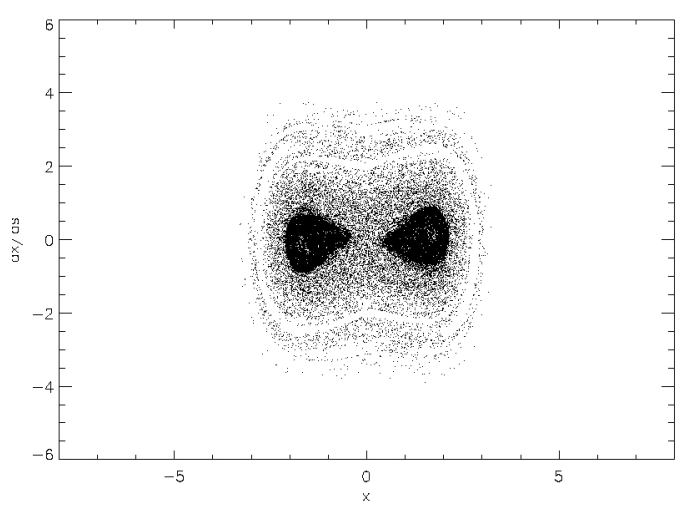

Figure 4: linear force, $\eta_{r m s}=0.31$

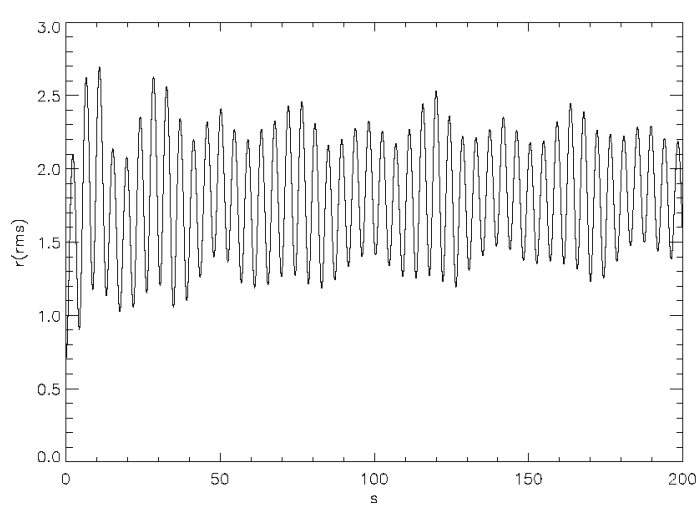

Figure 5: oscillation of the rms size of the beam for the $\eta_{r m s}=0.92$ case for linear focusing

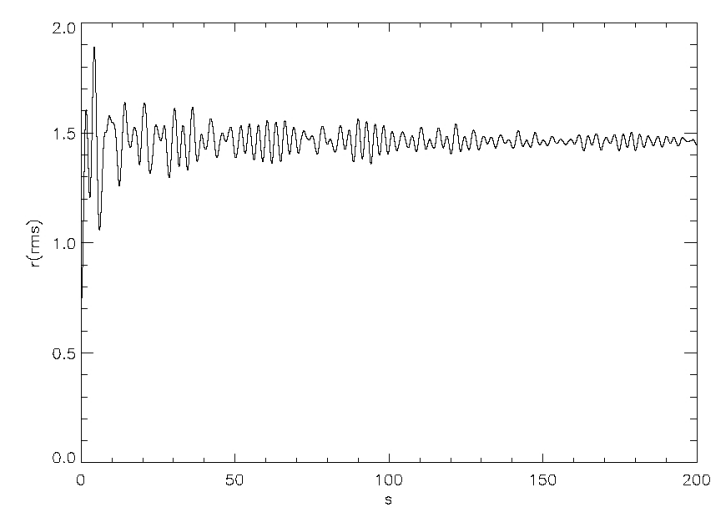

Figure 6: oscillation of the rms size of the beam for the $\eta_{r m s}=0.92$ case for nonlinear focusing

\section{CONCLUSION}

It can be clearly noticed that for both, the emittance and space charge dominated beams, particles do not drift far away from the core for the beams with nonlinear focusing. Thus we can conclude that the addition of nonlinear terms could mitigate beam halo formation. In order to better understand this phenomenon, we need to perform the simulations from an equilibrium distribution as mentioned in the previous section. This will be one of our future tasks.

\section{REFERENCES}

[1] R.L. Gluckstern, "Analytic Model for Halo Formation in High Current Ion Linacs", Phys. Rev. Lett., August 1994.

[2] R. L. Gluckstern et. al, "Halo Formation in ThreeDimensional Bunches", Phys. Rev. E, October 1998. 